\title{
Minimizing Risk of Nephrogenic systemic fibrosis in Cardiovascular Magnetic Resonance
}

\author{
Theresa Reiter ${ }^{1 *}$, Oliver Ritter ${ }^{1}$, Martin R Prince ${ }^{2}$, Peter Nordbeck${ }^{1}$, Christoph Wanner ${ }^{1}$, Eike Nagel ${ }^{3}$ and \\ Wolfgang Rudolf Bauer ${ }^{1}$
}

\begin{abstract}
Nephrogenic Systemic Fibrosis is a rare condition appearing only in patients with severe renal impairment or failure and presents with dermal lesions and involvement of internal organs. Although many cases are mild, an estimated $5 \%$ have a progressive debilitating course. To date, there is no known effective treatment thus stressing the necessity of ample prevention measures. An association with the use of Gadolinium based contrast agents (GBCA) makes Nephrogenic Systemic Fibrosis a potential side effect of contrast enhanced magnetic resonance imaging and offers the opportunity for prevention by limiting use of gadolinium based contrast agents in renal failure patients. In itself toxic, Gadolinium is embedded into chelates that allow its safe use as a contrast agent. One NSF theory is that Gadolinium chelates distribute into the extracellular fluid compartment and set Gadolinium ions free, depending on multiple factors among which the duration of chelates exposure is directly related to the renal function. Major medical societies both in Europe and in North America have developed guidelines for the usage of GBCA. Since the establishment of these guidelines and the increased general awareness of this condition, the occurrence of NSF has been nearly eliminated. Giving an overview over the current knowledge of NSF pathobiochemistry, pathogenesis and treatment options this review focuses on the guidelines of the European Medicines Agency, the European Society of Urogenital Radiology, the FDA and the American College of Radiology from 2008 up to 2011 and the transfer of this knowledge into every day practice.
\end{abstract}

\section{Review}

Cardiovascular Magnetic Resonance (CMR) has gained an increasingly important role among the diagnostic methods due to its superb soft tissue imaging qualities without ionizing radiation and minimal risks. CMR is one of the uprising areas of MRI offering deep insight in both cardiac structure and function which minimizes the risks of failing to make an accurate diagnosis or resorting to more invasive tests [1]. Use of contrast enhanced imaging techniques allows the assessment of perfusion, tissue viability and detailed angiographic studies. The overwhelming majority of these techniques use Gadolinium based contrast agents (GBCAs). From the early days of GBCA enhanced imaging, these contrast agents were considered safe with only rare allergic reactions or local irritation from extravasations. However, in 1997, a new disease emerged, originally called Nephrogenic Fibrosing

\footnotetext{
* Correspondence: Reiter_T@medizin.uni-wuerzburg.de

'Department of Internal Medicine I, Divisions of Cardiology and Nephrology,

University Hospital Wuerzburg, Wuerzburg, Germany

Full list of author information is available at the end of the article
}

Dermopathy. It was first described by Cowper in 2001 [2] and the relationship to GBCA exposure has been strongly suspected since 2006 [3-7]. Later this entity was renamed Nephrogenic Systemic Fibrosis (NSF) when involvement of internal organs was discovered. An NSF Registry, www. icnfdr.org documented over 355 cases that all occurred in patients on dialysis or with severe renal dysfunction [8]. However since the connection between NSF and GBCAs has become known changes in MRI protocols with the focus on prevention has led to a decrease in NSF incidence. Reports are showing virtually no new NSF cases since 2008 in both patients with normal renal function and patients with renal impairment [9-11] in spite of continued use of GBCA, albeit at lower doses.

Here we review the clinical features of NSF and show how to use GBCA safely in patients at risk for NSF.

\section{Pathobiochemistry of gadolinium}

Gadolinium is one of the 14 elements of the lanthanide group. Virtually all of its compounds contain it as the paramagnetic $\mathrm{Gd}^{3+}$ ion which has seven unpaired 
electrons in its half-filled $4 \mathrm{f}$ outer shell. $\mathrm{Gd}^{3+}$ has a long electronic relaxation time based on its totally symmetric $\mathrm{S}$ state making it well suited for use as an MR contrast agent. It accelerates the relaxation of the water molecules present in the tissue, giving rise to an enhanced signal on T1-weighted images and, together with appropriate sequence parameters, an improved image contrast. However, gadolinium, like most metals, interferes with the complex biochemical processes of living organisms. In particular, it can act as a competitive inhibitor of calcium ions due to its large ionic radius $(0.97 \AA$ vs. $1.06 \AA$ for $\mathrm{Ca}^{2+}$ ) and its high ionic charge. As a result various physiological processes involving $\mathrm{Ca}^{2+}$ can be influenced by the presence of $\mathrm{Gd}^{3+}$ such as $\mathrm{Ca}^{2+}$-activated ATPase in the sarcoplasmatic reticulum of skeletal muscle fibres, the reticuloendothelial system and some other enzymes such as dehydrogenases and glutathione $\mathrm{S}$ transferases. It is also known that $\mathrm{Gd}^{3+}$ has an inhibitory effect on Kupffer cells [4].

The toxic effects of $\mathrm{Gd}^{3+}$ can be suppressed by encasing it in an organic chelator. A variety of such chelators have been FDA/EMEA approved for use in patients and others are still being investigated [12-14]. The contrast agents in clinical use are based on the linear ionic chelator diethylenetriamine pentaacetic acid (often dubbed as DTPA or "pentetic acid"), the linear non-ionic chelator benzyloxyproprionictetra- acetate ("BOPTA") or on the cyclic ionic chelator tetraazacyclododecane tetraacetic acid (DOTA). Other cyclic non-ionic chelators are tetraazacycl ododecane (DO3A). Stability of these agents is characterized in two ways: Thermodynamic stability describes the tendency of the chelate to dissociate into its components given an unlimited amount of time. It is expressed numerically as the logarithm of the stability constant. Kinetic stability describes the timescale of the dissociation expressed either as a rate constant or a half-life. Both characteristics depend on the surrounding milieu: Decreasing $\mathrm{pH}$ and increasing temperature favour dissociation [15]. As a rule, the macrocyclic chelates are several orders of magnitude more stable with regard to dissociation and transmetalation than their linear counterparts [16-18]. Furthermore, the presence of ions such as $\mathrm{Ca}^{2+}, \mathrm{Cu}^{2+}$ or $\mathrm{Zn}^{2+}$ which can replace $\mathrm{Gd}^{3+}$ from the chelate in a transmetalation reaction promote the unwanted release of $\mathrm{Gd}^{3+}$ exposing tissues to its toxic effects [19]. Indeed, early in vivo studies using radioisotope labelled Gd chelates indicate a relationship between kinetic stability and tissue-uptake of gadolinium [20].

Besides the characterization in terms of macrocyclic and linear chelate structures, the nine currently available GBCAs (Table 1, Figure 1) can also be categorized by ionicity. Non-ionic $\mathrm{Gd}^{3+}$ chelates cause less osmotic stress because they do not require counterions such as $\mathrm{Na}^{+}$in their formulations. They are closer to ionic neutrality with lower viscosity, and are less hydrophilic than ionic $\mathrm{Gd}^{3+}$ chelates [21]. As with iodinated contrast, non-ionic $\mathrm{Gd}^{3+}$ chelates appear to have a lower rate of allergic reactions [22]. Unfortunately non-ionic linear chelates are also less stable than their negatively charged analogs. With the exception of three linear ionic GBCAs that have lipophilic groups in their chelate structures (Gadobenate, Gadoxetate, Gadofosvescet), GBCAs are eliminated exclusively via the renal pathway. The estimated half-life of renal elimination of GBCAs for patients with normal renal function is about $90 \mathrm{~min}$. However, with decreasing renal function the effective half-life increases to up to $18-34$ hours $[16,23]$. Within this timescale gadolinium release for the linear chelates may be significant [20].

Two of the three mentioned linear, ionic GBCAs (Gadobenate, Gadoxetate) have an aromatic component within the chelate structure that allows hepatocellular uptake and partial excretion via the biliary pathway. The third mentioned GBCA (Gadofosveset) has a biphenylcyclohexyl group that reversibly binds to albumin, extending the plasma half-life to about 18.5 hours for patients with a normal renal function $[16,19]$.

\section{Pathogenesis of nephrogenic systemic fibrosis}

NSF occurs in patients with acute or chronic renal failure $[32,33]$. The vast majority of NSF cases (approximately $95 \%$ ) have occurred in renal failure patients who received GBCA enhanced CMR imaging techniques prior to symptom onset [34]. Thus, it is likely that GBCAs play a role in triggering NSF. All GBCA stimulate the proliferation of fibroblasts, the linear GBCAs show a more potent stimulation than macrocyclic GBCAs [35-38] which raises the possibility that GBCA dissociation from the chelator is not necessary for NSF to occur. One hypothesis is that macrophages phagocytose the $\mathrm{Gd}^{3+}$ complexes that then, being located in intracellular lysosomes, stimulate the production of cytokines, and growth factors [39,40]. Local inflammation may be due to local $\mathrm{Gd}^{3+}$ deposition, that is triggered by local CD68+ or XIIIa+ dendritic cells, and a systemic inflammatory response that is associated with CD34+ fibrocytes that originate from the bone marrow [41]. The heterogeneous phenotypes of cells found in NSF lesion imply that both local and systemic inflammatory mechanisms might coexist [42]. TGF-beta1 levels are elevated in patients with NSF, and some studies have shown increased decortin levels, alpha-smooth muscle actin and hyaluron synthesis [38,43-45]. A modulation of collagen syntheses with an increase of Collagen I and III production as well as an increase in fibronectin expression has been documented $[38,46]$, as well as increased VEGF levels, Ostepontin, and TIMP-1 expression [47]. At least for one of the GBCAs (Gadodiamide), an effect on the expression of chemokine genes has been tracked down as the presence of 
Table 1 Stability of Gadolinium- based magnetic resonance imaging contrast agents in human serum at $37^{\circ} \mathrm{C}, \mathrm{K}($ therm)

\begin{tabular}{|c|c|c|c|c|c|c|}
\hline Class & Net Charge & Trade Name & Short Names & Log K(therm) & Log K(cond7.4) & Kinetic Stabiltiy \\
\hline \multirow[t]{6}{*}{ Linear } & Non-ionic & Omniscan & Gadodiamide & 16.9 & 14.9 & Low \\
\hline & Non-ionic & Optimark & Gadoversetamide & 16.8 & 15.0 & Low \\
\hline & Ionic & Magnevist & Gadopentetate & 22.5 & 18.4 & Medium \\
\hline & Ionic & Multihance & Gadobenate & 22.6 & 18.4 & Medium \\
\hline & Ionic & Primovist & Gadoxetate & 23.5 & 18.7 & Medium \\
\hline & Ionic & Vasovist & Gadofosvescet & 22.1 & 18.9 & Medium \\
\hline \multirow[t]{3}{*}{ Macrocyclic } & Non-ionic & Gadovist & Gadobutrol & 21.8 & 15.5 & High \\
\hline & Non-ionic & Prohance & Gadoteridol & 23.8 & 17.2 & High \\
\hline & Ionic & Dotarem & Gadoterate & 25.6 & 19.3 & High \\
\hline
\end{tabular}

Stability constants measured at high basic $\mathrm{pH}$. K(cond7.4) describe the complex stability constant at physiological conditions (pH 7.4). The values of K(cond7.4) are smaller than the values of $\mathrm{K}($ therm). Smaller values indicate less complex stability [24] [21]. The Values of K(therm) and K(cond7.4) are based on studies of Kumar, Cacheris, Toth, Imura, Schmitt-Willich, Uggeri, Caravan and Moreau [12,25-31]. Kinetic stability describes the activation energy required to break the chelateGadolinium bond.

GBCAs increase the activation of a NFkB pathway. It shows that exposure to GBCAs and the included $\mathrm{Gd}^{3+}$ is a potent stimulator of normal macrophages [48]. Most recent studies suggest that possibly other cells might be involved in the development of NSF as well, such as tissue monocytes and macrophages in the peripheral blood [49].

Additionally, it has been discussed that pro-inflammatory events, like vascular thrombosis, myocardial infarction, trauma, sepsis or recent surgery might contribute to the development of NSF [33]. Sepsis might even trigger the onset of NSF without exposure to GBCAs [50]. A resent work showed that the presence of GBCA increases DNA damage in lymphocytes [51]. The presence of high iron and erythropoetin levels are suspected to contribute to the development of NSF as well [44,52-54]. The $\mathrm{Gd}^{3+}$ complexes seem to have an effect on calcium phosphate as a study showed that the calcium phosphate precipitation is increased thus activating macrophages [55].

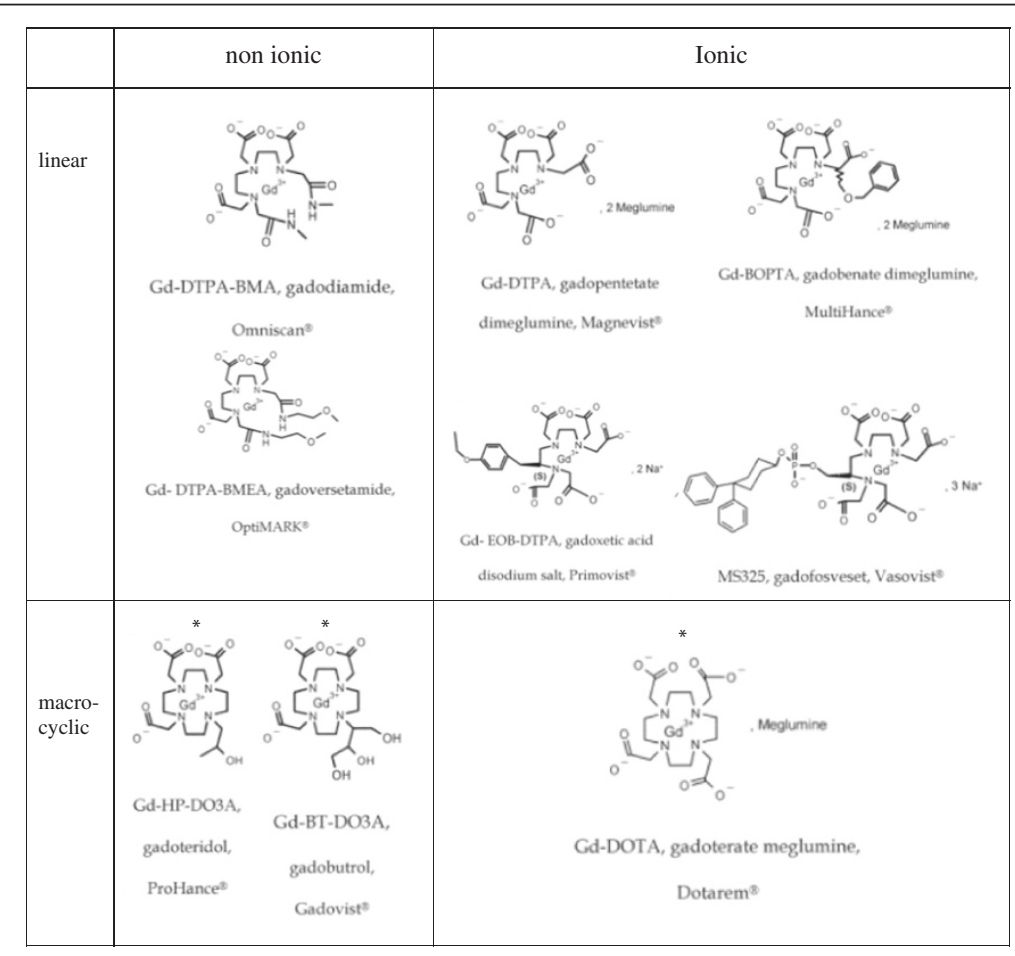

Figure 1 Molecular structures of all currently available Gadolinium- based contrast agents. The three agents in the lower section (asterisk) are presently considered the agents with highest safety due to their macrocyclic structure. 


\section{Symptoms, diagnosis and differential diagnosis}

Patients with NSF present with skin lesions typically beginning on the distal extremities starting with indurated plaques and papules especially on edematous lower extremities, later on the upper extremities, trunk and eye section develop over days to several weeks and later show a woody texture. The plaques are described as brawny, and the skin may develop hyperpigmentation [56] [57]. NSF lesions usually occur symmetrically. Patients may report sharp pain as well as pruritus, causalgias and paresthesias in afflicted areas. Stiffness and joint contractures can lead to decreased mobility. The progression is rapid in an estimated $5 \%$ of cases [8] leading to immobility within weeks and in a few instances death has been attributed to NSF. Besides skin developing lesions, internal organs can be afflicted including lung, heart, liver, bones and kidneys $[33,34]$.

NSF diagnosis is usually postulated upon the medical history/physical exam and confirmed with a deep punch skin biopsy $[2,56,58,59]$. Eosin and hematoxylin staining are used for demonstrating the typical features. The skin biopsy shows a dermal fibrosis with a high density of $C D$ 34 positive and procollagen I positive fibrocytes (circulating fibrocytes) and collagen bundles with prominent clefts between the bundles. Besides these collagen bundles elastic fibres can be detected as well. Additionally, but not required for diagnosis of NSF, factor XIIIa positive dendritic cells may be detected [56]. However the histopathological and clinical features can overlap with other entities. Among these are Lipodermatosclerosis, Scleroderma and Morphea, Scleromyxedema, Porphyria cutanea tarda, Spanish toxic Oil syndrome, eosinophilic fasciitis and Eosinophilia- myalgia syndrome and chronic graft versus host disease $[2,58,60]$. It is necessary to combine both the clinical features and the histopathologic findings in order to avoid misdiagnosis.

Exposure to gadolinium might arouse suspicion towards the diagnosis of NSF however it has to be stressed that exposure to GBCAs does not factor into the diagnosis. The lack of GBCA exposures does not exclude NSF as diagnosis, as in about $5 \%$ of NSF patients no GBCA exposure prior to symptom onset could be found.

Besides the development of NSF other more acute reactions to GBCA exposure have been described. Symptoms that imply the development of septicaemia have been described within 12-36 h after administration of GBCA [61]. Allergic reactions albeit rare are another GBCA risk [22] [62]. The possibility of deterioration in renal function after GBCA exposure in renal failure patients is controversial but in the usually administered dosages GBCAs are less nephrotoxic than iodinated contrast agents even with the high doses used for MR viability imaging and MR Angiography [63,64].

\section{Incidence and prevalence}

The incidence of NSF prior to 2008 varied widely among different institutions ranging from $0.26 \%$ in patients on dialysis without any contributing factors to up to $8.8 \%$ in patients with a eGFR smaller than $15 \mathrm{ml} / \mathrm{min} / 1.73 \mathrm{~m}^{3}$ without hemodialysis $[50,65]$. A study published in 2007 in another center calculated an absolute risk for developing NSF in patients on chronic dialysis of $2.4 \%$ per GBCA enhanced study and an absolute risk of $3.4 \%$ per patient [66]. The combination of renal insufficiency and proinflammatory processes (e.g. operations, thrombembolic events, endothelial/vascular injury) adds up to an NSF incidence of $4.6 \%$ [33]. The incidence is further increased when renal failure patients on dialysis develop sepsis, and has been estimated at $6.3 \%$ [50]. It is also noteworthy that the overwhelming majority of cases was either on dialysis or had an eGFR $<15 \mathrm{ml} / \mathrm{min} / 1,73 \mathrm{~m}^{3}$. Only a handful of cases estimated in patients with an estimated GFR $>30 \mathrm{ml} / \mathrm{min} /$ $1,73 \mathrm{~m}^{3}$ and most of these were patients in acute renal failure where GFR estimation is unreliable. The incidence of reported NSF cases in patients with a normal renal function is zero.

The vast majority of NSF cases have been reported in patients who underwent GBCA enhanced imaging however, about $5 \%$ of NSF cases showed no traceable exposure to GBCAs prior to NSF onset [34]. The exposure to GBCAs seems to be a cofactor to developing NSF, with the incidence depending besides the already mentioned patients collective also on the type and dose of GBCA. In patients who received a GBCA dose according to the labeling (e.g. $0.1 \mathrm{mmol} / \mathrm{kg}$ ) overall incidence is near zero, regardless of their renal function. Based on a report of Prince (2008, [65]) the use of higher GBCA doses such as double or triple dose GBCA

\section{Table 2 Overview of the examined treatment options}

\begin{tabular}{ll}
\hline Established therapies & Physical Therapy \\
& Pain control \\
& (opoids, NSARs, steroids, antidepressants) \\
& Renal Transplantation \\
Based on case reports & Extracorporal photopheresis \\
& Plasmapheresis \\
& UV- A1 therapy \\
& High dose iv. Immunoglobulines \\
& Imatinib Mesylate \\
& Rabamycine \\
& Pentoxifylline \\
& Topical dovonex \\
Cytoxan & Thalidomide \\
Immunosuppression via Steroids
\end{tabular}

The treatment is limited to symptomatic relief. 
administration $(0,2-0,3 \mathrm{mmol} / \mathrm{kg})$ increases the incidence from zero to $0,17 \%$ for some of the available contrast agents. These were Gadodiamide and Gadobenate, while this effect was not detected with Gadopentetate dimeglumine and Gadoteridol. The incidence rises when GBCAs in high doses are used in patients with renal failure. One of the reports describes the incidence in this setting at $8.0 \%[65,67-69]$.

The majority of reported cases are associated with non-ionic linear contrast agents (Gadodiamide or Gadoversetamide). Another agent associated with NSF is Gadopentate dimeglumine, but there are markedly fewer cases with Gadopentate dimeglumine than with Gadodiamide in spite of Gadopentetate dimeglumine having greater market share. Based on data presented at the FDA Joint Meeting of the Cardiovascular and Renal Drugs and Drug Safety Advisory Committee (Dec. 2009, observed time frame 2005-2009), 382 cases of NSF are related to the administration of Gadodiamide (estimated doses 13 million worldwide), 195 cases related to the administration of Gadopentetate dimeglumine (estimated doses 23 million doses worldwide) and 35 cases are attributed to Gadoversetamide (estimated 4.7 million doses worldwide). To date, no cases have been associated with the administration of Gadoxetate or Gadofosveset [70].
The American NSF registry has documented over 355 proven cases of NSF so far, however, other groups have reported deviant numbers, e.g. Zou et al. report of 408 cases that were biopsy confirmed [71]. Most likely, not all cases have been reported to the NSF registry, but directly to the FDA or not at all.

NSF is rarely seen in pediatric patients [72-74]. The youngest known case of NSF is a 6 year old patient even though many newborn babies with immature kidneys in the past received high doses of GBCA for multiple MR scans to assess congenital heart disease. This suggests that infants and newborns may be a protected population [63].

\section{Treatment of nephrogenic systemic fibrosis}

Many NSF patients have improved or even been cured with restoration of normal renal function. This has occurred when acute renal failure resolves and with renal transplantation. Otherwise, there is no proven effective therapy for the treatment of NSF and to date the treatment options are limited to symptomatic relief. Physical therapy supposedly improves the range of motion [75]. Pain medication usually is needed and includes the use of opioids, NSAR's, steroids and antidepressants. A single case reports that acetazolamide showed a good pain relieving effect in a meningeal affection of NSF. In some

Table 3 The FDA guidelines from 2006, 2007 and 2010 (www.fda.org)

\begin{tabular}{|c|c|c|}
\hline \multirow[t]{2}{*}{ FDA 2006} & FDA 2007 & FDA 2010 \\
\hline & Boxed Warning for GBCAs & Change in labeling for GBCAs \\
\hline Patients at risk: & Patients at risk: & Patients at risk: \\
\hline \multirow{4}{*}{$\begin{array}{l}\text { Moderate to end stage kidney disease } \\
\left(\mathrm{GFR}<60 \mathrm{ml} / \mathrm{min} / 1.73 \mathrm{~m}^{2}\right)\end{array}$} & \multirow{3}{*}{$\begin{array}{l}\text { Acute or chronic severe renal insufficiency } \\
\left(\mathrm{GFR}<30 \mathrm{ml} / \mathrm{min} / 1.73 \mathrm{~m}^{2}\right) \text { Acute renal } \\
\text { insufficiency due to hepatorenal } \\
\text { syndrome or in the perioperative phase } \\
\text { of a liver transplant }\end{array}$} & Highest risk for patients with GFR $<30 \mathrm{ml} / \mathrm{min} / 1.73 \mathrm{~m}^{2}$ \\
\hline & & Repeated or high dosage GBCA enhanced procedures \\
\hline & & NO RISK: patients with normal kidney function \\
\hline & $\begin{array}{l}\text { Screen all patients for renal dysfunction } \\
\text { (history and/or lab tests) }\end{array}$ & $\begin{array}{l}\text { Screen all patients for renal dysfunction } \\
\text { (history for assessment of acute kidney failure, } \\
\text { lab tests for patients at risk for chronic renal failure) } \\
\text { Risk factors are Diabetes, Hypertension, heart disease, } \\
\text { smoking, obesity, high cholesterol, family history of } \\
\text { kidney disease, age } 65 \text { or older, urinary tract } \\
\text { infections/obstructions, systemic infections } \\
\text { or autoimmune diseases [91] }\end{array}$ \\
\hline Measures: & Measures: & Measures: \\
\hline $\begin{array}{l}\text { Carefully weigh pros and cons of GBCA } \\
\text { enhanced imaging }\end{array}$ & avoid GBCA unless absolutely necessary & $\begin{array}{l}\text { Gadodiamide, Gadoversetamid, Gadopentet Acid } \\
\text { are contraindicated for highest risk patients }\end{array}$ \\
\hline \multirow[t]{2}{*}{ Use alternative imaging if possible } & do not exceed recommended dose & \\
\hline & $\begin{array}{l}\text { take the elimination half life into account } \\
\text { and allow enough time for GBCA elimination } \\
\text { before rescanning the patient }\end{array}$ & $\begin{array}{l}\text { Avoid GBCAs in patients with impaired } \\
\text { (known/suspected) drug elimination unless } \\
\text { absolutely necessary }\end{array}$ \\
\hline \multirow[t]{2}{*}{$\begin{array}{l}\text { Consider prompt dialysis in all patients } \\
\text { with impaired renal function }\end{array}$} & \multirow[t]{2}{*}{$\begin{array}{l}\text { Consider prompt dialysis in patients that are } \\
\text { already on dialysis treatment }\end{array}$} & $\begin{array}{l}\text { Consider prompt dialysis in patients that are } \\
\text { already on dialysis treatment }\end{array}$ \\
\hline & & $\begin{array}{l}\text { Monitor patients after GBCA administration, } \\
\text { good documentation, no repeat GBCA imaging } \\
\text { procedures until GBCA is eliminated from the body }\end{array}$ \\
\hline
\end{tabular}


Table 4 The EMA guidelines (www.ema.europa.eu)

\begin{tabular}{ll}
\hline $\begin{array}{l}\text { Patients with } \\
\text { severely } \\
\text { impaired renal } \\
\text { function }\end{array}$ & $\begin{array}{l}\text { high risk GBCAs are contraindicated } \\
\text { medium and low risk GBCAs: use lowest } \\
\text { possible dose, pause at least 7d between } \\
\text { two GBCA enhanced procedures }\end{array}$ \\
$\begin{array}{l}\text { Patients with } \\
\text { moderately } \\
\text { impaired renal } \\
\text { function }\end{array}$ & $\begin{array}{l}\text { High risk GBCAs: use single injection of } \\
\text { minimum dose, pause of 7d between } \\
\text { two GBCA enhanced procedures } \\
\text { Medium and low risk GBCAs: use minimum dose, } \\
\text { pause of 7d between two GBCA enhanced procedures }\end{array}$ \\
Toddlers $<1$ 1yo & $\begin{array}{l}\text { High, medium and low risk GBCAs: use single } \\
\text { injection of minimum dose, pause of 7d between } \\
\text { two GBCA enhanced procedures }\end{array}$ \\
Infants $<$ 4wks & $\begin{array}{l}\text { high risk GBCAs are contraindicated } \\
\text { medium and low risk GBCAs: use lowest possible } \\
\text { dose, pause at least 7d between two GBCA } \\
\text { enhanced procedures }\end{array}$ \\
Breast feeding & $\begin{array}{l}\text { High risk GBCAs: pause for 24 } \mathrm{h} \\
\text { Medium and low risk GBCAs: consider } \\
\text { pause for 24 } \mathrm{h}\end{array}$ \\
Perioperative & $\begin{array}{l}\text { high risk GBCAs are contraindicated } \\
\text { medium and low risk GBCAs: } \text { use lowest possible } \\
\text { dose, pause at least 7d between two GBCA } \\
\text { liver transplants }\end{array}$ \\
\hline enhanced procedures
\end{tabular}

cases intravenous procainamid has been shown to suppress the nociceptive aspects of pain. However, its application has to be performed under continuous cardiac monitoring.

The treatment options for NSF are based on case studies with limited numbers of patients. In the early days of NSF, the benefits of intense hemodialysis were discussed. In patients with end stage kidney disease approximately $98 \%$ of the Gadolinium that freely circulate in the blood is removed with 3 hemodialysis cycles. Not more than $30 \%$ of the Gadolinium chelates are removed with conventional dialysis techniques. Use of ultrapure dialysate and a high bicarbonate concentration combined with high flow for a duration of 5-6 h is said to achieve the best clearance rate [76]. There are some cases where a renal transplant and the resulting improved renal function lead to a decrease in the NSF symptoms. Among other discussed therapy options that were published are the extracorporal photopheresis, plasmapheris, UV-A1 Therapy, high dose intravenous Immunoglobulins, Imatinib Mesylate, Rabamycine and Pentoxifylline. The NSF registry additionally mentions some therapeutic regimens that were anecdotally reported, namely topical Dovonex, Cytoxan and Thalidomide. The use of oral steroids was discussed as well however the reports on the effectiveness were inconsistent (Table 2) [76-89].

Considering the small numbers of case reports and the fact that NSF can possibly be avoided by preventive measures, the focus of further attention should be primarily on prevention.

\section{Present guidelines in Europe and the USA - European Medicines Agency (2010), European Society of Urogenital Radiology (2008), FDA (2010) and American College of Radiology (2010)}

The FDA was the first agency to release a public health advisory back in 2006 when the association of GBCA and NSF was not as convincing. However the linkage of high dose GBCA with NSF was suspected, accordingly the advisory stated that only if deemed necessary patients with advanced kidney failure (dialysis or eGFR $<15 \mathrm{ml} / \mathrm{min} / 1.73 \mathrm{~m}^{2}$ ) should receive imaging studies

Table 5 Recommendations for specific patient groups by the American College of Radiology, 2010

\begin{tabular}{|c|c|}
\hline Chronic Kidney Disease Stage 1 or 2 & \multirow[t]{2}{*}{ No special measures } \\
\hline (eGFR 60 to $119 \mathrm{ml} / \mathrm{min} / 1.73 \mathrm{~m}^{2}$ ) & \\
\hline Chronic Kidney Disease Stage 3a & \multirow[t]{2}{*}{ Lowest possible dose of GBCA for diagnostic studies. If possible, avoid high risk GBCAs } \\
\hline (eGFR $45-59 \mathrm{ml} / \mathrm{min} / 1.73 \mathrm{~m}^{2}$ ) & \\
\hline Chronic Kidney Disease Stage 3b & \multirow{2}{*}{$\begin{array}{l}\text { GBCA use should be safe if dosage is restricted to } 0.1 \mathrm{mmol} / \mathrm{kg} \text { or less. } \\
\text { Apply same safety measures as in the following group }\end{array}$} \\
\hline (eGFR 30- $44 \mathrm{ml} / \mathrm{min} / 1.73 \mathrm{~m}^{2}$ ) & \\
\hline $\begin{array}{l}\text { Chronic Kidney Disease Stage } 4 \text { or } 5 \\
\text { not on chronic dialysis }\end{array}$ & \multirow[t]{2}{*}{$\begin{array}{l}\text { If feasible, avoid all contrast media. If usage is not avoidable, use I } \\
\text { owest possible dose, avoid high risk GBCAs, avoid readministration of GBCAs for a week }\end{array}$} \\
\hline$\left(\mathrm{eGFR}<30 \mathrm{ml} / \mathrm{min} / 1.73 \mathrm{~m}^{2}\right)$ & \\
\hline End-stage renal disease with chronic dialysis & \multirow{2}{*}{$\begin{array}{l}\text { Consider change of imaging modality. Avoid high risk GBCAs, use lowest possible dose. } \\
\text { Schedule the imaging study as close as possible before the next routine } \\
\text { hemodialysis session. }\end{array}$} \\
\hline (eGFR 45- 59 ml/min/1.73 m²) & \\
\hline Acute kidney injury & $\begin{array}{l}\text { If possible avoid any GBCA administration, regardless of eGFR. If GBCA use is necessary, } \\
\text { avoid high risk GBCAs, use lowest possible dose. }\end{array}$ \\
\hline Pregnancy & If possible, avoid use of GBCA, unless such an imaging study is absolutely necessary. \\
\hline Pediatrics & $\begin{array}{l}\text { Use similar safety measures as in adults. Neonates can have an eGFR of less than } \\
30 \mathrm{ml} / \mathrm{min} / 1.73 \mathrm{~m}^{3} \text { but is not considered an absolute contraindication. }\end{array}$ \\
\hline
\end{tabular}


using GBCA. An update in 2007 extended the patient collective at risk to patients with an eGFR of less than $30 \mathrm{ml} / \mathrm{min} / 1.72 \mathrm{~m}^{2}$ and also linked the induction of NSF to the hepato-renal syndrome and liver transplants. A screening of the renal function was proposed. GBCA dose should not exceed the FDA approved levels. In 2010 the latest FDA recommendations explicitly contraindicated Gadodiamide, Gadoversetamide and Gadopentetate dimeglumine use in patients with acute kidney disease or chronic severe kidney disease
$($ eGFR $<30)$. In general all GBCA should be avoided in patients with suspected or known impaired drug elimination unless the diagnostic GBCA based study is necessary. It is again stressed that the recommended dosage must not be exceeded and repeat dosing should be delayed until sufficient time has passed for the first dose to be completely eliminated. This might be less than one day for patients with normal renal function but up to a week for patients for severe chronic renal disease. The screening of the renal function and good

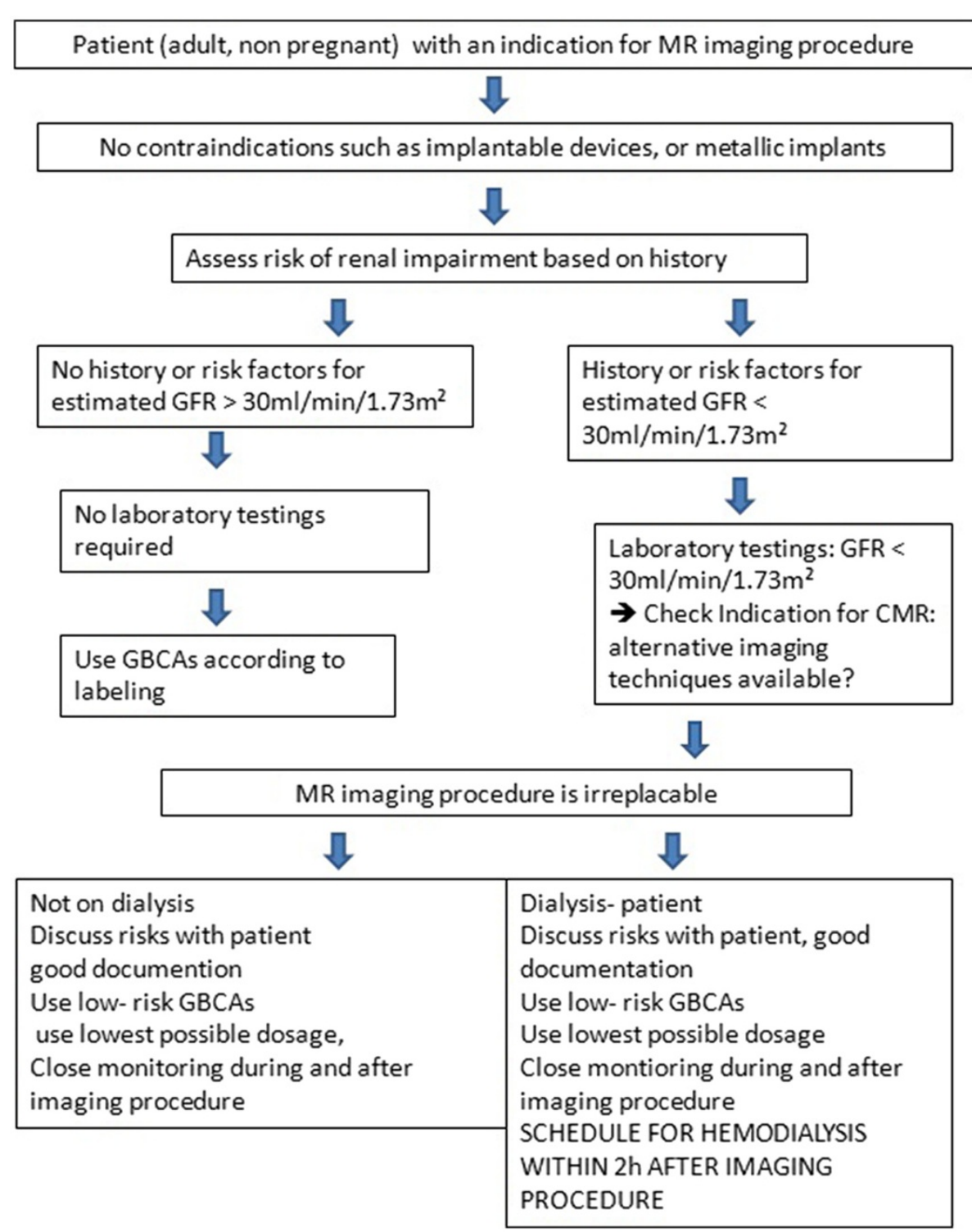

Figure 2 Work sheet "How to do it in adults"Work sheet "How to do it in adults". Among the risk factors for chronic kidney disease are Diabetes, Hypertension, heart disease, smoking, obesity, high cholesterol, racial factors, family history of kidney disease, ager 65 or older, urinary tract infections/obstructions, systemic infections or autoimmune diseases [91]. Standard doses for relevant procedures are 0.05-0.1 mmol/kg body weight for perfusion studies, $0.1-0.2 \mathrm{mmol} / \mathrm{kg}$ body weight for late gadolinium enhanced studies and 0.1-0.2 mmol/kg body weight for angiography studies $[100,101]$. 
documentation are also obligatory (Table 3) [90]. It should be also noted, that only little data on the safety of GBCAs for pediatric patients and almost no data for children younger than 2 years old is available.

The European Medicines Agency (EMA) has defined three risk classes of Gadolinium- based contrast agents. For each of them, the EMA has defined measures to reduce the risk of NSF.

\section{High risk}

Gadodiamide; Gadoversetamide, Gadopentetate

Dimeglumine

2. Intermediate risk

Gadobenate Dimeglumine; Gadoxetate Disodium;

Gadofosveset

3. Low risk

Gadobutrol, Gadoteridol; Gadoterate Meglumine

The EMEA's recommendations on the use of GBCAs are based on this classification (see Table 4). However the concern about newborns is controversial because there are no known cases of NSF in any patient less than 6 years old in spite of a extensive use of high doses of gadolinium based contrast agents for evaluating congenital heart disease in this population [63]. The guidelines also point to the necessity of good documentation of contrast agent type and dose [92] which has not been reliable in the past (Table 4) [93].

The European Society of Urogenital Radiology agrees with the EME on three risk classes for Gadolinium based contrast agents. Additionally, the ESUR defines three risk classes for patients. Patients with chronic kidney disease (CKD) stage 4 and 5 (eGFR $<30 \mathrm{ml} / \mathrm{min}$ ), patients on dialysis and patients with impaired renal function and pending or received liver transplant are considered high risk patients. Patients with CKD stage 3 and small children younger than 1 year old have a lower risk and with regards to renal function healthy patients are at no risk for developing NSF. The EUSR guidelines also mention the treatment of pregnant women. Due to lack of experience, these patients are to be treated according to the protocol for infants [94].

Further preventative measures should be strived for. It is theorized that low iron serum levels, and an optimized Calcium, phosphate and acid base balance before injection of GBCAs may protect against the development of NSF [85]. Recent research has focused on the goal to limit GBCA doses as far as possible in order to still ensure good image quality. Some authors have reported sufficient image quality in $3 \mathrm{~T}$ scanners e.g. for abdominal imaging with $0,025 \mathrm{mmol} / \mathrm{kg}$ GBCA at $3 \mathrm{~T}$ [95], 0,05 mmol/ $\mathrm{kg}$ for soft tissue characterization [96] and with $0,05 \mathrm{mmol} / \mathrm{kg}$ for vascular malformations [97]. Modifications in CE-MRA protocols offer further reduction of contrast agent doses [98].
Table 6 Current label doses for GBCAs [102]

\begin{tabular}{ll}
\hline Gadoteridol & $0.1 \mathrm{mmol} / \mathrm{kg}$ or $0.2 \mathrm{ml} / \mathrm{kg}$ \\
Gadoversetamide & $0.1 \mathrm{mmol} / \mathrm{kg}$ or $0.2 \mathrm{ml} / \mathrm{kg}$ \\
Gadodiamide & CNS: $0.1 \mathrm{mmol} / \mathrm{kg}$ or $0.2 \mathrm{ml} / \mathrm{kg}$ \\
& Body: $0.05 \mathrm{mmol} / \mathrm{kg}$ or $0.1 \mathrm{ml} / \mathrm{kg}$ \\
& $\begin{array}{l}\text { Intrathoracic/intraabdominal } / \mathrm{pelvic}: 0.1 \mathrm{mmol} / \mathrm{kg} \\
\text { or } 0.2 \mathrm{ml} / \mathrm{kg}\end{array}$ \\
& $0.1 \mathrm{mmol} / \mathrm{kg}$ or $0.2 \mathrm{ml} / \mathrm{kg}$ \\
$\begin{array}{l}\text { Gadobenate } \\
\text { dimeglumine }\end{array}$ & $0.1 \mathrm{mmol} / \mathrm{kg}$ or $0.2 \mathrm{ml} / \mathrm{kg}$ \\
$\begin{array}{l}\text { Gadopentetate } \\
\text { dimeglumine }\end{array}$ & $0.12 \mathrm{mmol} / \mathrm{kg}$ or $0.03 \mathrm{ml} / \mathrm{kg}$ \\
Gadofosveset & $0.025 \mathrm{mmol} / \mathrm{kg}$ or $0.1 \mathrm{ml} / \mathrm{kg}$ \\
Gadoxetate disodium & \\
Gadoterate meglumine $0.1 \mathrm{mmol} / \mathrm{kg}$ or $0.2 \mathrm{ml} / \mathrm{kg}$ \\
Gadobutrol
\end{tabular}

Guidelines of the American College for Radiology (2010) The American College of Radiology (ACR) recommendations of 2010 agree with the other guidelines on the associations of GBCA exposure, dosage relations and kidney function. The need for adequately assessing the renal function has been highlighted. In the opinion of the ACR patients at risk, e.g. patients with a past medical history of a renal disease, aged above 60 years old, who have hypertension or diabetes mellitus, should be tested for their renal function 6 weeks prior the planned CMR study. Additionally, a verbal assessment might be helpful. The recommendations for the specific patient groups, divided by their renal function is listed in Table 5 [99].

The recommendations of the above mentioned guidelines can be incorporated into an easy to use short work sheet for non- pregnant adults for everyday use (Figure 2, Table 6: list of current label doses).

\section{Conclusions}

NSF is one of the major risks associated with GBCA enhanced CMR procedures. Many of its features and pathogenetic characteristics have been already revealed, however important aspects still have to be focused on, for example the treatment of NSF. For the time being, the treatment is limited to symptomatic relieve and prevention. Several up to date guidelines are available, explaining the aspects of a safe GBCA enhanced CMR procedure. The benefits of GBCA enhanced CMR techniques are many in numbers, and should not be withheld rashly. Even with the lack of new NSF cases, the general awareness for NSF has to be strengthened in order to prevent the reoccurrence of this GBCA side effect.

\section{Competing interests}

MRP: patent agreements with Bayer, Bracco, GE Healthcare, Mallinckrodt, Lantheus and Epix. 
EN: Significant research support: Bayer Healthcare, all others have no conflicts of interest.

\section{Acknowledgement}

We thank Dr. W. A. Schenk, Institut fuer Anorganische Chemie, Univ. Wuerzburg, for supporting contributions.

\section{Author details}

${ }^{1}$ Department of Internal Medicine I, Divisions of Cardiology and Nephrology, University Hospital Wuerzburg, Wuerzburg, Germany. ${ }^{2}$ Department of Radiology, Cornell \& Columbia Universities, New York, USA. ${ }^{3}$ Division of Imaging Sciences, King's College London, London, UK.

\section{Authors' contributions}

TR carried out the review of literature, manuscript design and drafting. OR, MRP, PN, CW has been involved in revising the manuscript critically for important intellectual content. EN and WRB has been involved in drafting the manuscript and participated in manuscript design and revision. All authors read and approved the final manuscript.

Received: 14 February 2011 Accepted: 20 May 2012

Published: 20 May 2012

\section{References}

1. The European Cardiovascular Magnetic Resonance Registry. www.eurocmrregistry.com.

2. Cowper SE, et al: Nephrogenic fibrosing dermopathy. Am J Dermatopathol. 2001, 23(5):383-3.

3. Grobner T, Prischl FC: Gadolinium and nephrogenic systemic fibrosis. Kidney Int. 2007, 72(3):260-4.

4. Idee JM, et al: Possible involvement of gadolinium chelates in the pathophysiology of nephrogenic systemic fibrosis: a critical review. Toxicology. 2008, 248(2-3):77-8.

5. Marckmann P, et al: Nephrogenic systemic fibrosis: suspected causative role of gadodiamide used for contrast-enhanced magnetic resonance imaging. J Am Soc Nephrol. 2006, 17(9):2359-2.

6. Morcos SK: Gadolinium-based contrast agents and nephrotoxicity. Catheter Cardiovasc Interv. 2006, 68(5):812. author reply 813.

7. Thomsen HS, Morcos SK, Dawson P: Is there a causal relation between the administration of gadolinium based contrast media and the development of nephrogenic systemic fibrosis (NSF)? Clin Radiol. 2006, 61(11):905-6.

8. NSF Registry. www.pathmax.com/dermweb

9. Martin DR, et al: Decreased incidence of NSF in patients on dialysis after changing gadolinium contrast-enhanced MRI protocols. J Magn Reson Imaging. 2010, 31(2):440-6.

10. Rees O, Agarwal SK: Nephrogenic systemic fibrosis: UK survey of the use of gadolinium-based contrast media. Clin Radiol. 2010, 65(8):636-1.

11. Wang $Y$, et al: Incidence of Nephrogenic Systemic Fibrosis after Adoption of Restrictive Gadolinium-based Contrast Agent Guidelines. Radiology. 2011, 260(1):105-1.

12. Caravan P, et al: Thermodynamic stability and kinetic inertness of MS-325, a new blood pool agent for magnetic resonance imaging. Inorg Chem. 2001, 40(9):2170-6.

13. Gries H, Krause IW (Eds): Extracellular MRI Contrast Agents Based on Gadolinium Contrast Agents. Heidelberg: Springer Berlin; 2002:1-4.

14. Hermann P, et al: Gadolinium(III) complexes as MRI contrast agents: ligand design and properties of the complexes. Dalton Trans. 2008, 23:3027-7.

15. Brücher E: Kinetic Stabilities of Gadolinium(III) Chelates Used as MRI Contrast Agents. Top Curr Chem. 2002, 221:103-2.

16. Aime S, Caravan P: Biodistribution of gadolinium-based contrast agents, including gadolinium deposition. J Magn Reson Imaging. 2009, 30(6):1259-7.

17. Ersoy $H$, Rybicki FJ: Biochemical safety profiles of gadolinium-based extracellular contrast agents and nephrogenic systemic fibrosis. J Magn Reson Imaging. 2007, 26(5):1190-7.

18. Frenzel $\mathrm{T}$, et al: Stability of gadolinium-based magnetic resonance imaging contrast agents in human serum at 37 degrees $C$. Invest Radiol. 2008, 43(12):817-8.

19. Idee JM, et al: Role of thermodynamic and kinetic parameters in gadolinium chelate stability. J Magn Reson Imaging. 2009, 30(6):1249-8.
20. Wedeking P, Kumar K, Tweedle MF: Dissociation of gadolinium chelates in mice: relationship to chemical characteristics. Magn Reson Imaging. 1992, 10(4):641-8.

21. Port $M$, et al: Efficiency, thermodynamic and kinetic stability of marketed gadolinium chelates and their possible clinical consequences: a critical review. Biometals. 2008, 21(4):469-0.

22. Prince MR, et al: Incidence of Immediate Gadolinium Contrast Media Reactions. Am J Roentgenol. 2011, 196(2):W138-3.

23. Joffe $\mathrm{P}$, Thomsen HS, Meusel M: Pharmacokinetics of gadodiamide injection in patients with severe renal insufficiency and patients undergoing hemodialysis or continuous ambulatory peritoneal dialysis. Acad Radiol. 1998, 5(7):491-2.

24. Frenzel: Stability of Gadolinium- based magnetic resonance imaging contrast agents in human serum at $37^{\circ} \mathrm{C}$. 2008,

25. Cacheris WP, Quay SC, Rocklage SM: The relationship between thermodynamic and the toxicity of gadolinium complexes. Magn Reson Imaging 1990, 8(4):467-1.

26. Imura $H$, Choppin GR, Cacheris WP, de Learie LA, Dunn TJ, White DH: Thermodynamics and NMR studies of DTPA-bis(methoxyethylamide) and its derivatives. Protonation and complexation with Ln(III). Inorganica Chimica Acta. 1997, 258(2):227-6.

27. Kumar R, et al: Synthesis, in vitro biological stability, and anti-HIV activity of 5-halo-6-alkoxy(or azido)-5,6-dihydro-3'-azido-3'-deoxythymidine diastereomers as potential prodrugs to 3'-azido-3'-deoxythymidine (AZT). J Med Chem. 1994, 37(25):4297-6.

28. Moreau J, et al: Complexing mechanism of the lanthanide cations Eu3+, Gd3+, and Tb3+ with 1,4,7,10-tetrakis(carboxymethyl)-1,4,7,10tetraazacyclododecane (dota)-characterization of three successive complexing phases: study of the thermodynamic and structural properties of the complexes by potentiometry, luminescence spectroscopy, and EXAFS. Chemistry. 2004, 10(20):5218-2.

29. Schmitt-Willich $\mathrm{H}$, et al: Synthesis and Physicochemical Characterization of a New Gadolinium Chelate: The Liver-Specific Magnetic Resonance Imaging Contrast Agent Gd-EOB-DTPA. Inorg Chem. 1999, 38(6):1134-4.

30. Toth E, Kiraly R, Platzek J, Raduchel B, Brucher E: Equilibrium and kinetic studies on complexes of 10-[2,3-dihydroxy-(1-hydroxymethyl)-propyl]1,4,7,10-tetraazacyclododecane-1,4,7-triacetate. Inorganica Chimica Acta. 1996, 249(2):191-9.

31. Uggeri F, Uggeri FAS, Anelli P, Botta M, Brocchetta M, De Haen C, Ermondi G, Grandi M, Paoli P: Novel contrast agents for magnetic resonance imaging. Synthesis and characterization of the ligand BOPTA and its $\mathrm{Ln}(\mathrm{III})$ complexes ( $\mathrm{Ln}=\mathrm{Gd}, \mathrm{La}, \mathrm{Lu})$. X-ray structure of disodium (TPS-9-145337286-C-S)-[4-carboxy-5,8,11-tris(carboxymethyl)1-phenyl-2-oxa-5,8,11-triazatridecan-13-oato(5-)]gadolinate(2-) in a mixture with its enantiomer. Inorg Chem. 1995, 34:633-2.

32. Haylor J, et al: Nephrogenic gadolinium biodistribution and skin cellularity following a single injection of Omniscan in the rat. Invest Radiol. 2010, 45(9):507-2

33. Sadowski EA, et al: Nephrogenic systemic fibrosis: risk factors and incidence estimation. Radiology. 2007, 243(1):148-7.

34. Kuo PH, et al: Gadolinium-based MR contrast agents and nephrogenic systemic fibrosis. Radiology. 2007, 242(3):647-9.

35. Edward M, et al: Effect of different classes of gadolinium-based contrast agents on control and nephrogenic systemic fibrosis-derived fibroblast proliferation. Radiology. 2010, 256(3):735-3.

36. Morcos SK: Experimental studies investigating the pathophysiology of nephrogenic systemic fibrosis; what did we learn so far? Eur Radiol. 2011, 21(3):496-0

37. Tweedle MF, Wedeking $P$, Kumar K: Biodistribution of radiolabeled, formulated gadopentetate, gadoteridol, gadoterate, and gadodiamide in mice and rats. Invest Radiol. 1995, 30(6):372-0.

38. Varani J, et al: Effects of gadolinium-based magnetic resonance imaging contrast agents on human skin in organ culture and human skin fibroblasts. Invest Radiol. 2009, 44(2):74-1.

39. High WA, Ayers RA, Cowper SE: Gadolinium is quantifiable within the tissue of patients with nephrogenic systemic fibrosis. J Am Acad Dermatol. 2007, 56(4):710-2

40. High WA, et al: Gadolinium is detectable within the tissue of patients with nephrogenic systemic fibrosis. J Am Acad Dermatol. 2007, 56(1):21-6.

41. Mendoza FA, et al: Description of 12 cases of nephrogenic fibrosing dermopathy and review of the literature. Semin Arthritis Rheum. 2006, 35(4):238-9.

42. Quatresooz P, et al: Immunohistochemical aspects of the fibrogenic pathway in nephrogenic systemic fibrosis. Appl Immunohistochem Mol Morphol. 2010, 18(5):448-2. 
43. Bhagavathula $\mathrm{N}$, et al: Fibroblast response to gadolinium: role for plateletderived growth factor receptor. Invest Radiol. 2010, 45(12):769-7.

44. Gambichler T, et al: Decorin is significantly overexpressed in nephrogenic systemic fibrosis. Am J Clin Pathol. 2009, 132(1):139-3.

45. Pietsch $\mathrm{H}$, et al: The role of residual gadolinium in the induction of nephrogenic systemic fibrosis-like skin lesions in rats. Invest Radiol. 2011, 46(1):48-6.

46. Piera-Velazquez $\mathrm{S}$, et al: Persistent activation of dermal fibroblasts from patients with gadolinium-associated nephrogenic systemic fibrosis. Ann Rheum Dis. 2010, 69(11):2017-3.

47. Kelly $B C$, et al: The imbalanced expression of matrix metalloproteinases in nephrogenic systemic fibrosis. J Am Acad Dermatol. 2010, 63(3):483-9.

48. Del Galdo F, et al: NFkappaB activation and stimulation of chemokine production in normal human macrophages by the gadolinium-based magnetic resonance contrast agent Omniscan: possible role in the pathogenesis of nephrogenic systemic fibrosis. Ann Rheum Dis. 2010, 69(11):2024-3

49. Wiesinger $B$, et al: Effects of magnetic resonance imaging contrast agents on human umbilical vein endothelial cells and evaluation of magnetic resonance imaging contrast media-triggered transforming growth factorbeta induction in dermal fibroblasts (HSF) as a model for nephrogenic systemic fibrosis. Invest Radiol. 2011, 46(1):71-6.

50. Golding LP, Provenzale JM: Nephrogenic systemic fibrosis: possible association with a predisposing infection. AJR Am J Roentgenol. 2008, 190(4):1069-5

51. Yildiz S, et al: Impact of contrast enhanced MRI on lymphocyte DNA damage and serum visfatin level. Clin Biochem 2011, 44(12):975-9.

52. Hope TA, et al: Nephrogenic systemic fibrosis in rats treated with erythropoietin and intravenous iron. Radiology 2009, 253(2):390-8.

53. Schieren $G$, et al: Balance of profibrotic and antifibrotic [corrected] signaling in nephrogenic systemic fibrosis skin lesions. Am J Kidney Dis 2010, 55(6):1040-9.

54. Vakil $V$, et al: Gadolinium-containing magnetic resonance image contrast agent promotes fibrocyte differentiation. J Magn Reson Imaging 2009, 30(6):1284-8.

55. Gou BD, et al: Gadolinium-promoted precipitation of calcium phosphate is associated with profibrotic activation of RAW 264.7 macrophages. Toxicol In Vitro 2010, 24(6):1743-9.

56. Cowper SE, Rabach M, Girardi M: Clinical and histological findings in nephrogenic systemic fibrosis. Eur J Radiol. 2008, 66(2):191-9.

57. NSF Registry, updated. 2011. www.icnfdr.org.

58. Cowper SE: Nephrogenic fibrosing dermopathy: the first 6 years. Curr Opin Rheumatol. 2003, 15(6):785-0.

59. Samtleben W: Nephrogenic systemic fibrosis. Radiologe. 2007, 47(9):778-4

60. Cowper SE: Nephrogenic systemic fibrosis: an overview. J Am Coll Radiol. 2008, 5(1):23-8.

61. Steen $\mathrm{H}$, et al: Acute phase reaction to gadolinium-DTPA in dialysis patients. Nephrol Dial Transplant. 2009, 24(4):1274-7.

62. Brockow K, Ring J: Anaphylaxis to radiographic contrast media. Curr Opin Allergy Clin Immunol. 2011, 11(4):326-1.

63. Prince MR, et al: Risk factors for NSF: a literature review. J Magn Reson Imaging. 2009, 30(6):1298-8.

64. Prince MR, Arnoldus C, Frisoli JK: Nephrotoxicity of high-dose gadolinium compared with iodinated contrast. J Magn Reson Imaging. 1996, 6(1):162-6.

65. Prince MR, et al: Incidence of nephrogenic systemic fibrosis at two large medical centers. Radiology. 2008, 248(3):807-6.

66. Deo A, Fogel M, Cowper SE: Nephrogenic systemic fibrosis: a population study examining the relationship of disease development to gadolinium exposure. Clin J Am Soc Nephrol 2007, 2(2):264-7.

67. Collidge TA, et al: Gadolinium-enhanced MR imaging and nephrogenic systemic fibrosis: retrospective study of a renal replacement therapy cohort. Radiology 2007, 245(1):168-5.

68. Kallen $\mathrm{AJ}$, et al: Gadolinium-containing magnetic resonance imaging contrast and nephrogenic systemic fibrosis: a case-control study. Am J Kidney Dis. 2008, 51(6):966-5.

69. Marckmann $\mathrm{P}$, et al: Case-control study of gadodiamide-related nephrogenic systemic fibrosis. Nephrol Dial Transplant. 2007, 22(11):3174-8.

70. FDA - GBCA and NSF Core Presentations. http://www.fda.gov/downloads/ AdvisoryCommittees/CommitteesMeetingMaterials/Drugs/

CardiovascularandRenalDrugsAdvisoryCommittee/UCM196218.pdf. As seen on 1.Februar 2012
71. Zou Z, Ma L: Nephrogenic systemic fibrosis: review of 408 biopsyconfirmed cases. Indian J Dermatol. 2011, 56(1):65-3.

72. Auron A, Shao L, Warady BA: Nephrogenic fibrosing dermopathy in children. Pediatr Nephrol. 2006, 21(9):1307-1.

73. Foss $C$, et al: Gadolinium-associated nephrogenic systemic fibrosis in a 9year-old boy. Pediatr Dermatol. 2009, 26(5):579-2.

74. Jain SM, et al: Nephrogenic fibrosing dermopathy in pediatric patients. Pediatr Nephrol. 2004, 19(4):467-0.

75. Marckmann P: An epidemic outbreak of nephrogenic systemic fibrosis in a Danish hospital. Eur J Radiol. 2008, 66(2):187-0.

76. Silberzweig $\mathrm{Jl}$, Chung M: Removal of gadolinium by dialysis: review of different strategies and techniques. J Magn Reson Imaging. 2009, 30(6):1347-9.

77. Chung HJ, Chung KY: Nephrogenic fibrosing dermopathy: response to high-dose intravenous immunoglobulin. Br J Dermatol. 2004, 150(3):596-7.

78. Duffy KL, et al: Treatment of nephrogenic systemic fibrosis with Re-PUVA. J Am Acad Dermatol. 2008, 59(2 Suppl 1):S39-0.

79. Fretellier $\mathrm{N}$, et al: Clinical, biological, and skin histopathologic effects of ionic macrocyclic and nonionic linear gadolinium chelates in a rat model of nephrogenic systemic fibrosis. Invest Radiol. 2011, 46(2):85-3.

80. Geara AS, El-Imad B, El-Sayegh S: Acetazolamide therapy in meningeal involvement of nephrogenic systemic fibrosis. Intern Med J. 2010, 40(12):e4-5.

81. Kay J, High WA: Imatinib mesylate treatment of nephrogenic systemic fibrosis. Arthritis Rheum. 2008, 58(8):2543-8.

82. Marckmann P, Nielsen AH, Sloth JJ: Possibly enhanced Gd excretion in dialysate, but no major clinical benefit of 3-5 months of treatment with sodium thiosulfate in late stages of nephrogenic systemic fibrosis. Nephrol Dial Transplant. 2008, 23(10):3280-2.

83. Mathur $\mathrm{K}$, et al: Extracorporeal photopheresis improves nephrogenic fibrosing dermopathy/nephrogenic systemic fibrosis: three case reports and review of literature. J Clin Apher. 2008, 23(4):144-0.

84. Panesar M, et al: Transmetallation and gadolinium: Do low iron stores prevent the development of nephrogenic systemic fibrosis in high-risk end-stage renal disease patients? Hemodialysis Int. 2010, 14(3):289-4.

85. Perazella MA, Reilly RF: Imaging patients with kidney disease: how do we approach contrast-related toxicity? Am J Med Sci. 2011, 341(3):215-1.

86. Pieringer $\mathrm{H}$, et al: Treatment with corticosteroids does not seem to benefit nephrogenic systemic fibrosis. Nephrol Dial Transplant 2007, 22(10):3094.

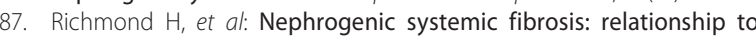
gadolinium and response to photopheresis. Arch Dermatol 2007. 143:(8) $1025-0$

88. Swaminathan $\mathrm{S}$, et al: Rapid improvement of nephrogenic systemic fibrosis with rapamycin therapy: possible role of phospho-70-ribosomalS6 kinase. J Am Acad Dermatol. 2010, 62(2):343-5.

89. Tran KT, et al: UV-A1 therapy for nephrogenic systemic fibrosis. Arch Dermatol. 2009, 145(10):1170-4.

90. U.S. Food and Drug Administration, www.fda.org, section drugs/drug safety.

91. Levey AS, et al: National Kidney Foundation practice guidelines for chronic kidney disease: evaluation, classification, and stratification. Ann Intern Med. 2003, 139(2):137-7.

92. European Medicines Agency EMA: Hauptinformationen für Bedienstete im Gesundheitswesen - Gadolinium- haltige Kontrastmittel und das Risiko für das Auftreten von nephrogenen systemischen Fibrosen (NSF). 2010. www.emea.org.

93. European Medicines Agency. www.ema.europa.eu/ema/, key words: Gadolinium based contrast agents.

94. European Society of Urogenial Radiology, ESUR: 2010, www.esur.org

95. De Campos ROP, et al: Quarter-Dose $(0.025 \mathrm{mmol} / \mathrm{kg})$ Gadobenate Dimeglumine for Abdominal MRI in Patients at Risk for Nephrogenic Systemic Fibrosis: Preliminary Observations. Am J Roentgenol. 2011, 196(3):545-2.

96. Costelloe $\mathrm{CM}$, et al: Comparison of half-dose and full-dose gadolinium MR contrast on the enhancement of bone and soft tissue tumors. Skeletal Radiol. 2011, 40(3):327-3.

97. Anzidei M, et al: Low-dose contrast-enhanced time-resolved MR angiography at $3 \mathrm{~T}$ : diagnostic accuracy for treatment planning and follow-up of vascular malformations. Clin Radiol. 2011, 66(12):1181-2.

98. Nael K. Moriarty JM. Finn JP. Low dose CE-MRA. Eur J Radiol, 2011, 80(1):2-8.

99. American College of Radiology - ACR: Subcommittee on MR Safety: ACR Manual on Contrast Media, Version 7. 2010

100. Kramer CM, et al: Standardized cardiovascular magnetic resonance imaging (CMR) protocols, society for cardiovascular magnetic resonance: 
board of trustees task force on standardized protocols. J Cardiovasc Magn Reson. 2008, 10:35.

101. Ishida $M$, et al: Development of a universal dual-bolus injection scheme for the quantitative assessment of myocardial perfusion cardiovascular magnetic resonance. J Cardiovasc Magn Reson. 2011, 13:28.

102. Informatin on drugs to be found on www.fda.org (US Food and Drug administration), http://www.drugs.com/pro/vasovist.html (Drug Information Online), www.medicines.org.au/files/aspdotar.pdf (Medicines Information Australia); http://www.primovist.com/scripts/pages/en/primovist/index.php (Bayer Product Information); http://www.gadovist.ch/de/gadovist/dosierunganwendung/ (Bayer Product Information).

doi:10.1186/1532-429X-14-31

Cite this article as: Reiter et al:: Minimizing Risk of Nephrogenic systemic fibrosis in Cardiovascular Magnetic Resonance. Journal of Cardiovascular Magnetic Resonance 2012 14:31.

\section{Submit your next manuscript to BioMed Central and take full advantage of:}

- Convenient online submission

- Thorough peer review

- No space constraints or color figure charges

- Immediate publication on acceptance

- Inclusion in PubMed, CAS, Scopus and Google Scholar

- Research which is freely available for redistribution 